\title{
Nota Científica \\ Emergência de plântulas e crescimento inicial de copaíba sob diferentes substratos
}

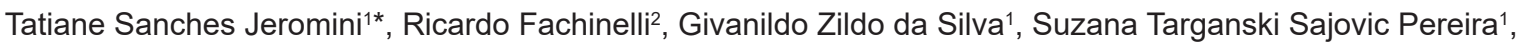
Silvana de Paula Quintão Scalon²

${ }^{1}$ Universidade Estadual Paulista “Julio de Mesquita Filho”, Via de Acesso Prof. Paulo Donato Castelane, s/n, Vila Industrial, CEP 14884-900, Jaboticabal, SP, Brasil ${ }^{2}$ Universidade Federal da Grande Dourados, Unidade 2, Rod. Dourados - Itahum, Km 12, Cidade Universitaria, CEP 79804-970, Dourados, MS, Brasil

"Autor correspondente:

tatiane_jeromini@hotmail.com

Termos para indexação:

Cerrado

Copaifera langsdorffi

Reflorestamento

Index terms:

Cerrado

Copaifera langsdorffi

Afforestation

\section{Histórico do artigo:}

Recebido em 18/03/16

Aprovado em 16/04/17

Publicado em 30/06/17

doi: 10.4336/2017.pfb.37.90.1189
Resumo - O objetivo do trabalho foi avaliar a emergência e crescimento inicial de mudas de copaíba (Copaifera langsdorffi Desf.) sob diferentes substratos. A semeadura

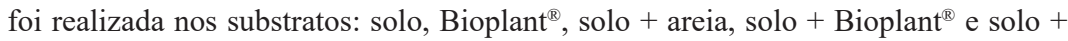
areia + cama-de-frango. Foram avaliadas emergência, velocidade de emergência e crescimento de plântulas. Para a emergência de plântulas de copaíba são indicados todos os substratos avaliados, exceto mistura solo + areia + cama-de-frango. Para o crescimento inicial, indicado pelo índice de qualidade de Dickson, o melhor substrato foi apenas solo.

\section{Emergence and initial growth of copaiba seedlings under different substrates}

\begin{abstract}
The aim of this study was to evaluate emergence and early growth of copaiba seedlings (Copaifera langsdorffi Desf.) under different substrates. Seeds were sown in substrates: soil, Bioplant ${ }^{\circledR}$, soil + sand, soil + Bioplant $^{\circledR}$ and soil + sand + chicken manure. Emergency, emergency speed and seedlings growth were evaluated. For copaiba seedlings emergency all evaluated substrates were indicated, except the mix soil + sand + chicken manure. For initial growth, indicated by Dickson quality index, the most suitable substrate was soil.
\end{abstract}

O cerrado brasileiro é composto por um mosaico de fisionomias que variam de campos abertos a florestas fechadas (Scalon et al., 2014). Em meio a esse hot spot, se destaca a espécie copaíba (Copaifera langsdorffii Desf. - Leguminosae - Caesalpinioidea).

O Brasil é o maior produtor e exportador do óleo de copaíba extraído do tronco (Masson et al., 2013), que tem amplo e tradicional emprego medicinal como antiflamatório, antisséptico (Santana et al., 2014) e agente antifúngico (Souza et al., 2013). A copa da árvore fornece sombra aos animais silvestres, em época seca, sendo útil para plantio em áreas degradadas e arborização urbana (Pereira, 2011). Entretanto, informações sobre a formação de mudas ainda são incipientes.

No processo de produção de mudas, o substrato interfere diretamente na qualidade das plantas resultantes, devido à variação das propriedades físicas, químicas e biológicas do mesmo, que pode afetar a germinação e o estabelecimento das plântulas nesta fase (Silva et al., 2011).

A escolha do substrato deve basear-se, entre outros fatores, no custo e na disponibilidade, de forma que 
várias misturas são sugeridas para a produção de mudas de espécies florestais, tais como o solo acrescido de areia para produção de mudas de guavira (Campomanesia adamandium) (Dresch et al., 2016); Bioplant ${ }^{\mathbb{B}}$ para canafístula, (Peltophorum dubium) (Dutra et al., 2012) e Latossolo + areia + cama de frango para uvaia (Eugenia pyriformis) (Scalon et al., 2014), dentre outras.

Pesquisas referentes à emergência de espécies nativas do cerrado ainda são limitadas. Assim no presente trabalho objetivou-se avaliar a emergência de plântulas e crescimento inicial de copaíba em diferentes substratos.

$\mathrm{O}$ estudo foi realizado na Universidade Federal da Grande Dourados, Dourados, MS. As sementes de copaíba foram coletadas de 10 matrizes localizadas na

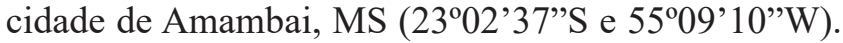
Foi retirado o arilo das sementes, sendo selecionadas as íntegras e livres de injúrias causadas por insetos ou doenças.

A semeadura foi realizada em tubetes de $50 \mathrm{~mm}$ x 190 $\mathrm{mm}$, com volume de $120 \mathrm{~cm}^{3}$, dispostos em bandejas de polipropileno tipo caixa $(620 \mathrm{~mm} \times 420 \mathrm{~mm} \times 165 \mathrm{~mm})$ com 54 células, em estufa com tela de sombreamento de $50 \%$, irrigadas para se manter $60 \%$ da capacidade de campo de cada substrato calculado pela fórmula de Souza et al. (2000). Foram estudados os seguintes substratos: solo (S), Bioplant ${ }^{\circledR}(\mathrm{B})$, solo + areia $(\mathrm{S}+\mathrm{A})$ (1:1), solo + Bioplant ${ }^{\mathbb{}}(\mathrm{S}+\mathrm{B})(1: 1)$ e solo + areia + camade-frango semidecomposta $(\mathrm{S}+\mathrm{A}+\mathrm{CF})(1: 1: 1)$.

O solo foi coletado na região de Dourados, MS, local de ocorrência da espécie em estudo, sendo classificado como Latossolo Vermelho Distroférrico de textura argilosa (Santos et al., 2013). A areia utilizada apresentava granulometria fina (partículas entre 0,10 e $0,25 \mathrm{~mm})$. O substrato Bioplant ${ }^{\circledR}$ utilizado é composto por casca de pinus, fibra de coco, vermiculita, macro e micronutrientes, que não são divulgados pelo fabricante, com pH entre 5,2 e 6,5 e condutividade elétrica entre 0,6 e $1,4 \mu \mathrm{S} \mathrm{cm}^{-1}$. A cama de frango semidecomposta estava em torno de 60 dias em fermentação, fase de humificação do material orgânico. A análise química dos substratos está apresentada na Tabela 1 .

Tabela 1. Análise química dos substratos avaliados para a emergência de plântulas e produção de mudas de copaíba.

\begin{tabular}{lccccccccc}
\hline \multirow{2}{*}{ Substrato } & $\mathbf{p H}$ & $\mathbf{P}$ & $\mathbf{K}$ & $\mathbf{C a}$ & $\mathbf{M g}$ & $\mathbf{H}+\mathbf{A l}$ & $\mathbf{L}$ & $\mathbf{C T C}$ & $\mathbf{V}$ \\
& $\mathbf{C a C l}_{2}$ & $\mathbf{m g d m}^{-3}$ & $\mathbf{m m o l}_{\mathbf{c}} \mathbf{d m}^{-3}$ & \multicolumn{2}{c}{$\mathbf{c m o l}_{\mathbf{c}} \mathbf{d m}^{-3}$} & & & $\mathbf{c m o l}_{\mathbf{c}} \mathbf{d m}^{-3}$ & $\mathbf{\%}^{-3}$ \\
\hline $\mathrm{S}$ & 4,9 & 16,7 & 50,0 & 4,1 & 2,0 & 5,5 & 6,7 & 6,7 & 54,8 \\
$\mathrm{~S}+\mathrm{A}$ & 4,4 & 0,6 & 30,0 & 1,4 & 0,6 & 1,8 & 19,9 & 3,8 & 52,0 \\
$\mathrm{~S}+\mathrm{B}$ & 5,2 & 51,0 & 39,3 & 10,5 & 5,6 & 7,8 & 19,93 & 27,8 & 71,9 \\
$\mathrm{~S}+\mathrm{A}+\mathrm{CF}$ & 6,5 & 230,9 & 30,1 & 2,9 & 2,6 & 1,5 & 8,54 & 10,0 & 84,9 \\
$\mathrm{~B}$ & 5,3 & 252 & 32,8 & 17,4 & 10,4 & 4,3 & 31,08 & 35,4 & 88 \\
\hline
\end{tabular}

Onde: $\mathrm{pH}$ em $\mathrm{CaCl}_{2}$ - $\mathrm{pH}$ em solução centimolar de cloreto de cálcio; $\mathrm{P}$ - fósforo extraído do solo por meio de Mehlich; $\mathrm{K}, \mathrm{Ca}$ e $\mathrm{Mg}$-formas trocáveis dos elementos; $\mathrm{H}+\mathrm{Al}$ - acidez potencial; $\mathrm{L}$ - soma de bases; $\mathrm{CTC}$ - capacidade de troca de cátions; $\mathrm{V} \%$ - índice de saturação por bases. S - solo; B- Bioplant ${ }^{\mathbb{E}} ; \mathrm{S}+\mathrm{A}$ - solo + areia; $\mathrm{S}+\mathrm{B}$ - solo + Bioplant $^{\mathbb{E}}$ e $\mathrm{S}+\mathrm{A}+\mathrm{CF}$ - solo + areia + cama-de-frango semidecomposta.

No sexagésimo terceiro dia avaliou-se a porcentagem de emergência $(\% \mathrm{E})$, o índice de velocidade de emergência (IVE), calculado pela fórmula de Maguire (1962), e o tempo médio de emergência (TME), obtido pela fórmula proposta por Labouriau (1983).

Os parâmetros de crescimento inicial avaliados foram: comprimento da parte aérea, (CPA) mensurado do coleto, até a inserção das últimas folhas apicais, comprimento de raiz (CR), mensurado da coifa até o coleto e diâmetro do coleto (DC), determinado com paquímetro digital. Avaliou-se também o teor de clorofila (TC), mensurado com clorofilômetro SPAD 502, obtendo-se uma média de três leituras para cada repetição, número de folhas (NF), massa fresca da parte aérea (MFPA) e das raízes (MFR), massa seca da parte aérea (MSPA), das raízes (MSR) e total (MST) e índice de qualidade de Dickson (IQD), obtido pela fórmula proposta por Dickson et al. (1960), conforme equação 1. 


$$
I Q D=\frac{M S T}{\frac{M S P A}{M S R}+\frac{C P A}{D C}}
$$

Onde: MST $=$ massa seca total; MSPA = massa seca da parte aérea; $\mathrm{MSR}=$ massa seca das raízes; $\mathrm{CPA}=$ comprimento de parte aérea e $\mathrm{DC}=$ diâmetro do coleto.

O delineamento estatístico adotado foi inteiramente casualizado com quatro repetições de 25 sementes cada. Os dados foram submetidos ao teste de normalidade de Shapiro-Wilk e à análise de variância, e as médias comparadas pelo teste de Tukey a $5 \%$ de probabilidade, sendo utilizado o software Sisvar 5.1 Build 72 (Ferreira, 2007).

Tabela 2. Porcentagem de emergência (E), índice de velocidade de emergência (IVE) de plântulas, número de folhas (NF), índice de qualidade de Dickson (IDQ), massa fresca de parte aérea (MFA) e de raiz (MFR), massa seca de parte aérea (MSA), de raízes (MSR) e total (MST) de mudas de copaíba, em função de diferentes substratos.

\begin{tabular}{lccccccccc}
\hline \multicolumn{1}{c}{ Substrato } & E & IVE & NF & IQD & MFA & MFR & MAS & MSR & MST \\
\hline $\mathrm{S}$ & $53 \mathrm{a}$ & $0,53 \mathrm{a}$ & $14 \mathrm{a}$ & $0,21 \mathrm{a}$ & $1,28 \mathrm{a}$ & $0,50 \mathrm{a}$ & $1,20 \mathrm{a}$ & $0,44 \mathrm{a}$ & $1,33 \mathrm{a}$ \\
$\mathrm{S}+\mathrm{A}$ & $53 \mathrm{a}$ & $0,56 \mathrm{a}$ & $10 \mathrm{~b}$ & $0,09 \mathrm{~cd}$ & $0,96 \mathrm{~d}$ & $0,36 \mathrm{ab}$ & $0,50 \mathrm{bc}$ & $0,18 \mathrm{bc}$ & $1,00 \mathrm{bc}$ \\
$\mathrm{S}+\mathrm{B}$ & $51 \mathrm{a}$ & $0,56 \mathrm{a}$ & $14 \mathrm{a}$ & $0,11 \mathrm{bc}$ & $1,07 \mathrm{bc}$ & $0,46 \mathrm{ab}$ & $0,67 \mathrm{~b}$ & $0,26 \mathrm{bc}$ & $1,08 \mathrm{~b}$ \\
$\mathrm{~S}+\mathrm{A}+\mathrm{CF}$ & $9 \mathrm{~b}$ & $0,24 \mathrm{~b}$ & $11 \mathrm{ab}$ & $0,07 \mathrm{~d}$ & $0,98 \mathrm{~cd}$ & $0,29 \mathrm{~b}$ & $0,42 \mathrm{c}$ & $0,14 \mathrm{c}$ & $0,96 \mathrm{c}$ \\
$\mathrm{B}$ & $66 \mathrm{a}$ & $0,56 \mathrm{a}$ & $11 \mathrm{ab}$ & $0,14 \mathrm{c}$ & $1,10 \mathrm{ab}$ & $0,53 \mathrm{a}$ & $0,69 \mathrm{~b}$ & $0,30 \mathrm{~b}$ & $1,09 \mathrm{~b}$ \\
\hline
\end{tabular}

Valores seguidos pela mesma letra minúscula na linha não diferem entre si pelo teste de Tukey ao nível de 5\% de probabilidade. S - solo; BBioplant ${ }^{\mathbb{\circledR}} ; \mathrm{S}+\mathrm{A}$ - solo + areia; S+B - solo + Bioplant $^{\circledR}$ e S+A+CF - solo + areia + cama-de-frango semidecomposta.

A presença de resíduos orgânicos, como a cama-defrango, na composição do substrato, pode ter afetado de forma negativa a absorção de água e de oxigênio pelas sementes nas fases iniciais da germinação, fazendo com que o processo germinativo ocorresse com menor velocidade. A germinação, segundo Marcos Filho (2015), pode ser afetada por diversos fatores, como os fatores do ambiente, como: água, temperatura, oxigênio, luz e promotores químicos. Em vista disso, maiores porcentagens de material orgânico presentes nos substratos podem ter influenciado no tempo necessário para a emergência das plântulas (Souza et al., 2001), devido à aeração, menor retenção de umidade, composição química e manutenção da temperatura durante o período de embebição das sementes.

Esse comportamento da germinação de sementes na presença de substratos com compostos orgânicos também foi relatado por Nogueira et al. (2012). Segundo esses autores, a medida que aumentava a proporção de composto orgânico na composição do substrato havia a redução da emergência de plântulas de Mimosa caesalpiniifolia mais acentuada. Souza (2010) também verificou que a emergência plântulas de Calotropis procera foi altamente prejudicada quando utilizou altas concentrações de composto orgânico.

Os substratos que proporcionaram maiores valores para o número de folhas (NF) foram $\mathrm{S}$ e $\mathrm{S}+\mathrm{B}$ (Tabela 2). Estes substratos contêm macro e micronutrientes de forma equilibrada em sua composição (Tabela 1). Os nutrientes presentes no substrato costumam ser assimilados pelas raízes da planta, possibilitando maior desenvolvimento foliar de modo similar ao verificado em Eugenia dysenterica (Silva et al., 2011), Sicana odorifera (Lima et al., 2010), E. uniflora (Antunes et al., 2012) e $S$. parahyba (Martins et al., 2012). 
Os substratos que proporcionaram melhores resultados para o índice de qualidade de Dickson (IQD) foram o S, seguido de $\mathrm{S}+\mathrm{B}$ (Tabela 2). Estes resultados sugerem que não é possível generalizar o efeito benéfico da adição de resíduos orgânicos na produção de mudas e que os resultados podem ser contraditórios quando se trabalha com uma espécie com silvicultura não domesticada, como é o caso da copaíba. Estudos com diversas espécies nativas do cerrado tem comprovado a existência de uma alta variabilidade genética dentro da mesma espécie, dificultado em alguns casos a padronização de técnicas de cultivos comerciais (Franzon, 2009; Ribeiro \& Rodriguez, 2006).

Além disso, de acordo com Paiva Sobrinho et al. (2010) e Silva \& Moraes (2013), espécies nativas do cerrado não respondem em crescimento e desenvolvimento com a adição de nutrientes minerais ou de matéria orgânica nos substratos, pois originalmente são adaptadas a solos de baixa fertilidade.

Para massa fresca e seca das plantas (Tabela 2), verificou-se que, de maneira geral, mudas oriundas do substrato solo apresentaram maiores massas, não diferindo do Bioplant ${ }^{\circledR}$ para massa fresca da parte aérea, tendo sido observada diferença de massa de raízes apenas para o tratamento com cama-de-frango. Esse foi o menos favorável para o acúmulo de massa seca e fresca, tanto da parte aérea como de raízes. Exceto na massa fresca da parte aérea que o substrato composto por solo e areia resultou nos menores valores.

Menor acúmulo de massa seca em função da adição de esterco também foi observada em mudas de guanandi (Calophyllum brasiliense), com adição de esterco ao substrato (Artur et al., 2007), e em mudas de olho-decabra (Ormosia arborea), com terra e esterco bovino (Silva \& Moraes, 2013).

Os resultados de massa seca mostram que Copaifera langsdorffi pode se desenvolver satisfatoriamente em solo desprovido de quaisquer fontes de matéria orgânica, com capacidade de utilizar apenas os nutrientes disponíveis no solo de origem. Paiva Sobrinho et al. (2010), ao testarem substratos na produção de mudas de Hancornia speciosa, Dipteryx alata, Eugenia dysenterica, que são espécies nativas do cerrado, também obtiveram maiores valores de massa seca com substrato contendo apenas solo (Argissolo Amarelo Eutrófico - CTC de $16,38 \mathrm{cmol}_{\mathrm{c}} \mathrm{dm}^{-3}$ ), mostrando que a fertilidade presente no solo foi suficiente para suprir as necessidades de crescimento da muda. No entanto, é importante considerar que, apesar de muitas espécies ocuparem o mesmo bioma e estarem submetidas a condições semelhantes, certas especificidades e exigências podem ser diferenciadas (Scalon \& Jeromine, 2013).

Os compostos orgânicos presentes no substrato solo + areia + cama-de-frango semidecomposta possuem um alto teor de fósforo em sua composição, quando comparados com os outros substratos (Tabela 1). Segundo Deon (2007) esse excesso pode provocar deficiência de micronutrientes na planta, como zinco e cobre, além de macronutrientes, como cálcio, pois reduzem sua absorção, interferindo de forma negativa no desenvolvimento das plantas.

A copaíba, por ser uma espécie nativa do Cerrado brasileiro onde os solos se caracterizam pela elevada intemperização e altos teores de $\mathrm{Al}^{+3} \mathrm{e}^{+}$, demonstrou que o tratamento constituído apenas de solo foi mais adequado para a fase de crescimento inicial das mudas. Entretanto, quando se avalia a extração de solo para uso como substrato é importante avaliar sua disponibilidade e uso racional deste recurso.

Salienta-se ainda que o uso do substrato solo é viável (Chagas Junior et al., 2013), pois mesmo com disponibilidade de vários substratos comerciais, não há conhecimento sobre as exigências nutricionais da maioria das espécies florestais nativas, acrescentandose ainda o fato de que o emprego desses produtos está restrito à produção em grande escala de mudas ou a determinadas regiões do país. Diante disto, uma opção para diminuir a quantidade de solo utilizada poderia ser a incorporação de Bioplant ${ }^{\circledR}$, pois de acordo com os resultados (Tabela 2) foi o segundo melhor substrato para produção de mudas dessa espécie.

Substratos alternativos ou misturas destes devem ser estudados, visando baratear os custos de produção e tornar o viveirismo atividade acessível a todos os interessados em recompor áreas degradadas ou explorar alguma atividade silvicultural (Chagas Junior et al., 2013).

\section{Conclusão}

Para a emergência de plântulas de copaíba são indicados todos os substratos avaliados, exceto o substrato solo + areia + cama-de-frango semidecomposta.

Para o crescimento inicial, indicado pelo índice de qualidade de Dickson, o melhor substrato é o composto apenas por solo. 


\section{Referências}

Antunes, L. E. C. et al. Influência do substrato, tamanho de sementes e maturação de frutos na formação de mudas de pitangueira. Revista Brasileira de Fruticultura, v. 34, n. 4, p. 1216-1223, 2012. DOI: 10.1590/S0100-29452012000400031.

Artur, A. G. et al. Esterco bovino e calagem para formação de mudas de guanandi. Pesquisa Agropecuária Brasileira, v. 42, n. 6, p. 843850, 2007. DOI: 10.1590/S0100-204X2007000600011.

Chagas Junior, A. F. et al. Desenvolvimento inicial de canafístula (Peltophorum dubium) em solos de cerrado no estado do Tocantins. Revista Verde de Agroecologia e Desenvolvimento Sustentável, v. 8, n. 5, p. 69-75, 2013.

Deon, M. D. Crescimento e nutrição mineral da soja submetida a excesso de P, S, K, Ca e Mg em solução nutritiva. $2007.71 \mathrm{f}$. Dissertação (Mestrado em Solos e Nutrição de Plantas) - Escola Superior de Agricultura "Luiz de Queiroz", Universidade de São Paulo, Piracicaba.

Dickson, A. et al. Quality appraisal of white spruce and white pine seedling stock in nurseries. Forest Chronicle, v. 36, p.10-13, 1960.

Dresch, D. M. et al. Initial growth of Campomanesia adamantium (Cambess.) O. Berg. seedlings on substrates with different compositions and water retention capacities. Bioscience Journal, v. 32, n. 1, p. 1-10, 2016. DOI: 10.14393/BJ-v32n1a2016-26121.

Dutra, T. R. et al. Emergência e crescimento inicial da canafístula em diferentes substratos e métodos de superação de dormência. Revista Caatinga, v. 25, n. 2, p. 65-71, 2012.

Ferreira, D. F. Sisvar: sistema de análise de variância para dados balanceados, versão 5.1 Build 72. Lavras: DEX/ UFLA, 2007.

Franzon, R. C. Fruteiras nativas do Cerrado têm potencial para exploração. Ecos do Tocantins, 01 dez. 2009.

Labouriau, L. G. A germinação das sementes. Lima: Secretaria Geral da OEA, 1983. 174 p. (OEA-Serie de Biologia. Monografia, 24).

Lima, J. F. et al. Avaliação de diferentes substratos na qualidade fisiológica de sementes de melão de caroá [Sicanaodorifera (Vell.) Naudim]. Revista Brasileira de Plantas Medicinais, v. 12, n. 2, p. 163-167, 2010.

Maguire, J. D. Speed of germination aid in selection and evaluation for seedling emergence and vigor. Crop Science, v. 2, n. 2, p. 176177, 1962.

Marcos Filho, J. Fisiologia de sementes de plantas cultivadas. 2. ed. Londrina: ABRATES, 2015. 660 p.

Martins, C. C. et al. Posição da semente na semeadura e tipo de substrato sobre a emergência e crescimento de plântulas de Schizolobium parahyba (Vell.) S.F. Blake. Ciência Florestal, v. 22, n. 4, p. 849-856, 2012.

Masson, D. S. et al. Atividades antimicrobianas do óleo-resina de copaíba (Copaifera langsdorffii) em bactérias de significância clínica em úlceras cutâneas. Revista Brasileira de Plantas Medicinais, v. 15, n. 4, p. 664-669, 2013. DOI: 10.1590/S151605722013000500006 .
Nogueira, N. W. et al. Emergência e desenvolvimento inicial de plântulas de Mimosa caesalpiniifolia Benth. em função de diferentes substratos. Revista Agroambiente On-line, v. 6, p. 17-24, 2012.

Paiva Sobrinho, S. et al. Substratos na produção de mudas de três espécies arbóreas do Cerrado. Revista Brasileira de Ciências Agrárias, v. 5, n. 2, p. 238-243, 2010. DOI: 10.5039/agraria. v5i2a741.

Pereira, M. D. S. Manual técnico: conhecendo e produzindo sementes e mudas da caatinga. Fortaleza: Associação Caatinga, 2011.

Ribeiro, R. A. \& Rodrigues, F. M. Genética da conservação em espécies vegetais do cerrado. Revista de Ciências Médicas e Biológicas, v. 5, n. 3, p. 253-260, 2006.

Santana, S. R. et al. Uso medicinal do óleo de copaíba (Copaifera sp.) por pessoas da melhor idade no município de Presidente Médici, Rondônia, Brasil. Acta Amazônica, v. 63, n. 4, p. 361-366, 2014. DOI: 10.15446/acag.v63n4.39111.

Santos, H. G. dos et al. Sistema brasileiro de classificação de solos. 3. ed. Brasília, DF: Embrapa, 2013. 353 p.

Scalon, S. P. Q. \& Jeromine, T. S. Substratos e níveis de água no potencial germinativo de sementes de uvaia. Revista Árvore, v. 37 , n. 1, 2013. DOI: 10.1590/S0100-67622013000100006.

Scalon, S. P. Q. et al. Photosynthetic metabolism and quality of Eugenia pyriformis Cambess. seedlings on substrate function and water levels. Anais da Academia Brasileira de Ciências, v. 86, n. 4, p. 2040-20148, 2014. DOI: 10.1590/0001-3765201420130249.

Silva, A. L. \& Morais, G. A. Influência de diferentes substratos no crescimento inicial de Ormosia arborea (Vell.) Harms (Fabaceae). Revista Verde de Agroecologia e Desenvolvimento Sustentável, v. 8, n. 4, p. 22-27, 2013.

Silva, E. A. et al. Substratos na produção de mudas de mangabeira em tubetes. Pesquisa Agropecuária Tropical, v. 41, n. 2, p. 279-285, 2011. DOI: 10.5216/pat.v41i2.9042.

Souza, B. C. M. et al. Atividade antifúngica de óleos vegetais artesanais e industrializados sobre fitopatógenos de espécies florestais. Cadernos de Agroecologia, v. 8, n. 2, 2013.

Souza, C. C. et al. Avaliação de métodos de determinação de água disponível e manejo da irrigação em solo roxa sob cultivo de algodoeiro herbáceo. Revista Brasileira de Engenharia Agrícola Ambiental, v. 4, p. 338-342, 2000. DOI: 10.1590/S141543662000000300006.

Souza, D. C. F. Crescimento e desenvolvimento inicial de Plântulas de flor de seda (Calotropis procera) em diferentes substratos. 2010. 32 f. Monografia (Graduação em Agronomia) Universidade Federal Rural do Semi-Árido, Mossoró.

Souza, E. R. B. et al. Emergência e crescimento de cagaita (Eugenia dysenterica DC em função do tipo e do volume de substratos. Pesquisa Agropecuária Tropical, v. 31, n. 2, p. 89-95, 2001.

Pesq. flor. bras., Colombo, v. 37, n. 90, p. 219-223, abr./jun. 2017 
